wherever safely possible. We however, recommend caution as model for sub-tariff CBE endoscopy from static sites needs to be first piloted.

Disclosure of Interest None Declared.

\section{OC-041 REDUCED HOSPITAL ADMISSION AND RAPID ACCESS TO SPECIALIST SERVICES THROUGH THE INTRODUCTION OF A GASTROENTEROLOGY AND HEPATOLOGY AMBULATORY CARE SERVICE}

1J Fielding ${ }^{*},{ }^{1} \mathrm{~A}$ Hawley, ${ }^{1} \mathrm{~S}$ Hardcastle, ${ }^{1} \mathrm{~K}$ Drew, ${ }^{2} \mathrm{D}$ Gleeson, ${ }^{2} \mathrm{M}$ Karajeh, ${ }^{1} \mathrm{D}$ Sanders, ${ }^{1}$ A Hopper, ${ }^{1} \mathrm{R}$ Sidhu, ${ }^{1} \mathrm{M}$ McAlindon, ${ }^{1} \mathrm{AJ}$ Lobo. ${ }^{1}$ Gastroenterology, Sheffield Teaching Hospitals NHS Foundation Trust, Sheffield, UK; ${ }^{2}$ Hepatology, Sheffield Teaching Hospitals NHS Foundation Trust, Sheffield, UK

\subsection{6/gutjnl-2014-307263.41}

Introduction Ambulatory care sensitive conditions are those where intervention may limit hospital admissions. Improvements in the management of these conditions may save the NHS $£ 96-$ $£ 238$ million per annum and alleviate pressures on Accident and Emergency. This is directly applicable in Gastroenterology but few gastrointestinal (GI) conditions are conventionally listed as suitable for this approach. This study examined the effect of such a service providing rapid access to specialist services in a UK Gastroenterology Unit.

Methods A Gastroenterology ambulatory care service was established as part of an investigation unit with on-ward endoscopy facilities. General practitioners (GPs) were given written guidelines and referred to a senior nurse via telephone. Patients considered suitable were those requiring urgent assessment but where admission might be avoided. Exclusion criteria: hypotension, suspected acute abdomen, or GP concern about potential for deterioration.

Results 224 patients were referred by their GP from June 2011 to January 2013. 12 did not attend.

Presentation and outcome are described in Table 1. 179 patients $(84 \%)$ were seen on arrival by a consultant. 96 patients (45\%) were admitted; 116 were discharged on the same day of whom 94 (91\%) were offered either same day $(n=67$ $(58 \%))$ or outpatient $(\mathrm{n}=27(23 \%))$ investigations. 30 day readmission rate was only $4 \%(n=5)$.

51 patients had low risk GI bleeds (Rockall score 0-1). 30 $(59 \%)$ of these were discharged the same day and $90 \%(\mathrm{n}=27)$ had an OGD within $24 \mathrm{~h}$ of assessment, either same day or returning the following morning.

Conclusion The Ambulatory Care Service provides direct, rapid access to specialist opinion and investigation for a range of GI presentations, avoiding hospital admission for the majority referred. In contrast to other UK studies those with low risk GI bleeding were managed as out-patients but with gastroscopy undertaken. This is a model for a tiered approach to emergency care in Gastroenterology.

Disclosure of Interest None Declared.

\section{OC-042 SENSITIVITY OF ANNUAL FAECAL IMMUNOCHEMICAL TESTS FOR HAEMOGLOBIN (FIT) FOR DETECTING ADVANCED NEOPLASIA IN PATIENTS UNDERGOING THREE-YEARLY SURVEILLANCE COLONOSCOPY - THE FIT FOR FOLLOW-UP STUDY}

${ }^{1} \mathrm{E}$ MacRae*, ${ }^{1} \mathrm{~A}$ Brenner, ${ }^{1} \mathrm{~J}$ Martin, ${ }^{2} \mathrm{~S}$ Pearson, ${ }^{2} \mathrm{C}$ Piggott, ${ }^{3} \mathrm{H}$ Bowyer, ${ }^{3} \mathrm{G}$ Vart, ${ }^{3} \mathrm{C}$ von Wagner, ${ }^{3} \mathrm{~J}$ Wardle, ${ }^{2,4} \mathrm{~S}$ Halloran, ${ }^{1}$ W Atkin. ${ }^{1}$ Imperial College, London, UK; ${ }^{2} \mathrm{NHS}$ $B C S P$, Guildford, UK; ${ }^{3} U C L$, London, UK; ${ }^{4}$ University of Surrey, Guildford, UK

\subsection{6/gutjin-2014-307263.42}

Introduction With increasing demand for colonoscopy in the NHS Bowel Cancer Screening Programme (BCSP) in England, there is a need for effective non-colonoscopic approaches to surveillance. We have undertaken a study to compare the sensitivity and specificity of a faecal immunochemical test for haemoglobin (FIT) annually for three years with colonoscopy surveillance, in patients diagnosed with intermediate-risk adenomas following a positive faecal occult blood test in the BCSP.

Methods Participants are invited to complete a FIT (OCSENSOR, Eiken Chemical Co. Ltd.) annually while awaiting their first surveillance colonoscopy. Those testing positive (>40 $\mu \mathrm{g} \mathrm{Hb} / \mathrm{g}$ faeces) are invited to have their three-year colonoscopy brought forward, while those testing negative are sent another FIT a year later until they have completed three rounds of testing. We aim to determine the sensitivity of FIT in detecting colorectal cancer or advanced adenomas $(\geq 10 \mathrm{~mm}$, or with tubulovillous or villous histology, or with high-grade dysplasia), using colonoscopy as the reference standard.

Results We invited 8009 people to participate in the study, of whom $5840(72.9 \%)$ consented. The positivity rate in Round 1 was 5.8\% (336/5840). To date, 265/303 (87.5\%) have had an early colonoscopy: 62/265 (23.4\%) had advanced adenomas and five $(1.9 \%)$ had cancer. $33 / 336 \quad(9.8 \%)$ declined an early colonoscopy.

To date, in Round 2 we have invited 2800 patients who tested FIT negative in Round 1: 2560 (91.4\%) completed a second FIT and 115/2560 (4.5\%) tested positive. 85/106 (80.2\%) have received an early colonoscopy: 13/85 (15.3\%) had

Abstract OC-041 Table 1 Summary of admissions and discharges

\begin{tabular}{|c|c|c|c|c|c|c|}
\hline$\underline{\text { Diagnosis }}$ & Number & Admitted & Discharged & $\begin{array}{l}\text { Discharged: same day } \\
\text { investigation } \\
\text { (\% of discharged) }\end{array}$ & $\begin{array}{l}\text { Discharged: OP } \\
\text { investigation } \\
\text { (\% of discharged) }\end{array}$ & $\begin{array}{l}\text { Discharged: } \\
30 \text { day readmit }\end{array}$ \\
\hline GI Bleed & 51 & $21(41 \%)$ & $30(59 \%)$ & $25(83 \%)$ & $2(7 \%)$ & 0 \\
\hline PR bleed & 16 & $8(50 \%)$ & $8(50 \%)$ & $4(50 \%)$ & $3(37.5 \%)$ & 0 \\
\hline Anaemia & 18 & $9(50 \%)$ & $9(50 \%)$ & $3(33 \%)$ & $4(44 \%)$ & 0 \\
\hline Abdo pain & 36 & $16(44 \%)$ & $20(56 \%)$ & $9(45 \%)$ & $8(40 \%)$ & $2(10 \%)$ \\
\hline IBD flare & 30 & $14(47 \%)$ & $16(53 \%)$ & $8(50 \%)$ & $2(12 \%)$ & 0 \\
\hline Deranged LFTs/ jaundice & 20 & $9(45 \%)$ & $11(55 \%)$ & $6(55 \%)$ & $3(27 \%)$ & $1(9 \%)$ \\
\hline Diarrhoea/vomiting & 15 & $5(33 \%)$ & $10(67 \%)$ & $5(50 \%)$ & $3(30 \%)$ & $1(10 \%)$ \\
\hline Other & 26 & $14(54 \%)$ & $12(46 \%)$ & $7(58 \%)$ & $2(17 \%)$ & $1(8 \%)$ \\
\hline Total & 212 & $96(45 \%)$ & $116(55 \%)$ & $67(58 \%)$ & $27(23 \%)$ & $5(4 \%)$ \\
\hline
\end{tabular}


advanced adenomas and two (2.4\%) had cancer. 9/115 (7.8\%) declined an early colonoscopy.

A total of 2445 participants had negative FIT tests in Rounds 1 and 2 and will be invited to complete a final FIT, prior to their surveillance colonoscopy.

Satisfaction with the study was high among those who completed a questionnaire, with 95.4\% (4177/4378) of FIT-negative and 91\% (203/223) of FIT-positive participants in Round 1 stating that they would complete another kit in future.

Conclusion Compliance with the study was high, and the majority of participants reported that they would use FIT again. Almost a quarter (23.4\%) of patients in Round 1 who had an early colonoscopy had advanced adenomas, falling to $15.3 \%$ in Round 2. Round 2 is ongoing, with Round 3 starting in January 2014.

Disclosure of Interest None Declared.

\section{Colorectal and anorectal free papers}

\section{OC-043 HOW COMMONLY IS COLORECTAL CANCER LATER DIAGNOSED FOLLOWING A COLONOSCOPY THAT DOES NOT REPORT COLORECTAL CANCER (AN ANALYSIS OF 11 YEARS OF NATIONAL DATA IN ENGLAND)?}

${ }^{1} \mathrm{D}$ Cheung*, ${ }^{2} \mathrm{~F}$ Evison, ${ }^{3} \mathrm{P}$ Patel, ${ }^{1} \mathrm{~N}$ Trudgill. ${ }^{1}$ Department of Gastroenterology, Sandwell General Hospital, West Bromwich, UK; ${ }^{2}$ Health Informatics Department, Queen Elizabeth Hospital, Birmingham, UK; ${ }^{3}$ School of Cancer Sciences, University of Birmingham, Birmingham, UK

\subsection{6/gutjnl-2014-307263.43}

Introduction Colonoscopy is the standard of care for diagnosing colorectal cancer (CRC). However, 3.4\%-7.9\% of subjects with $\mathrm{CRC}$ are reportedly diagnosed within $3 \mathrm{yrs}$ of a colonoscopy that did not detect the cancer (post-colonoscopy colorectal cancer, PCCRC). We have investigated risk factors for these events in a national data set in England.

Methods Hospital Episode Statistics (HES) collates information on all NHS hospital attendances in England. Subjects undergoing colonoscopy without a CRC diagnosis 6-36 months before subsequent CRC diagnosis were identified as PCCRC cases (definitely missed - colonoscopy without CRC diagnosis 6-12 months before CRC diagnosis; probably missed - colonoscopy without CRC diagnosis 12-36 months before CRC diagnosis) and those with no colonoscopy 6-36 months before diagnosis served as controls. The influence of personal and institutional variables on missed PCCRC were examined by multivariate logistic regression.

Results HES records from 2001-12 were analysed including 2874641 colonoscopies in 2263905 subjects. 136237 subjects were diagnosed with CRC. 4219 (3.1\%) definitely missed PCCRC cases and 8266 (6.1\%) probably missed PCCRC cases occurred. Colonic polyps were the most common coded finding in PCCRC subjects (1553 subjects (12.4\%)). Emergency colonoscopies were less likely to fail to diagnose CRC than elective procedures (OR 0.58 (95\% CI: 0.5-0.6), p < 0.001). Subjects over age $70(1.16(1.1-1.2), \mathrm{p}<0.001)$, female gender $(1.05(1.0-$ $1.1), \mathrm{p}=0.018)$ and comorbidities (liver disease (2.18 (1.4$3.5), \mathrm{p}=0.002)$, peptic ulcer $(1.29(1.1-1.6), \mathrm{p}=0.01)$, myocardial infarction $(1.14(1.0-1.3), \mathrm{p}=0.046)$, pulmonary disease (1.11 (1.0-1.2), p = 0.025)) were associated with PCCRC. Ethnicity was not associated with PCCRC. Right sided CRC was more likely to be missed (1.30 (1.25-1.37), $\mathrm{p}=0.015)$. Subjects with PCCRC were less likely to undergo surgery $(0.27(0.26-$ $0.28), \mathrm{p}=<0.001)$ or chemotherapy $(0.62(0.59-0.65), \mathrm{p}=$ $<0.001)$. Overall survival was worse in PCCRC subjects than controls. There was a fourfold variation in PCCRC rates between units. Unit volume was inversely related to PCCRC rate (lowest tertile volume versus highest tertile 1.72 (1.6-1.8), $\mathrm{p}=$ $<0.001)$. The annual rate of PCCRC has improved over the study period with a fall in PCCRC rate from 15.9 to $5.1 \%$ per annum.

Conclusion The rate of PCCRC up to 3 yrs prior to CRC diagnosis was 9.1\% in England between 2001-12. PCCRC was associated with increasing age, female gender, comorbidities, site in right colon and colonoscopy unit volume. PCCRC subjects were less likely to have surgery or chemotherapy and had worse survival rates. Encouragingly, annual rates of PCCRC have fallen over the study period.

Disclosure of Interest None Declared.

\section{OC-044 PREDICTORS OF ADVANCED NEOPLASIA AT SURVEILLANCE IN SCREENING POPULATION- A STUDY OF ALL HIGH AND INTERMEDIATE RISK GORUP SUBJECTS IN FIRST SIX YEAR OF NHS BCSP}

${ }^{1} \mathrm{D}$ Majumdar ${ }^{*},{ }^{2} \mathrm{AP}$ Hungin, ${ }^{2} \mathrm{DW}$ Wilson, ${ }^{3} \mathrm{C}$ Nickerson, ${ }^{1} \mathrm{MD}$ Rutter. ${ }^{1}$ Gastroenterology, North Tees Hospital, Stockton on Tees, UK; ${ }^{2}$ Wolfson's Research Institute, Durham University, Stockton on Tees, UK; ${ }^{3}$ NHS Cancer Screening Service, Sheffield, UK

\subsection{6/gutjil-2014-307263.44}

Introduction In NHS BCSP, high and intermediate risk subjects with colorectal adenomas undergo surveillance colonoscopies. This guideline evidence was derived mostly from general population based studies. This study aims to evaluate the individual and adenoma specific characteristics detected at the index colonoscopy which can predict occurrence of advanced neoplasia during surveillance in a well-defined FOB screening population. Methods The national BSCP database was interrogated to identify all subjects who participated during the period of June 2006 to July 2012 and completed their first surveillance. The subjects where all the adenomas were retrieved during screening colonoscopy were included. Multivariate analysis was performed to identify the factors which determine occurrence of CRC and advanced adenoma (AA = adenoma with size $\geq 10 \mathrm{~mm} />25 \%$ villous histology/ high grade dysplasia) at surveillance.

Results A total of 17694 high and intermediate risk subjects participated, and 7015 of them completed their first surveillance and were included for analysis. The adenoma specific factors evaluated were high grade dysplasia, villous histology $\geq 25 \%$, and size $\geq 10 \mathrm{~mm}$, number of adenomas and any proximal location of adenoma at screening. The individual characteristic

\begin{tabular}{lll} 
Abstract OC-044 Table 1 & & \\
\hline Predictor factor & AA OR $(95 \% \mathrm{Cl})$ & p-AA \\
\hline Male gender & $1.39(1.07-1.8)$ & 0.01 \\
Proximal Location & $1.8(1.3-2.6)$ & $<0.001$ \\
Adenoma size $\geq 10 \mathrm{~mm}$ & $1.01(0.7-1.36)$ & 0.93 \\
5 I > adenomas & $2.4(1.6-3.4)$ & $<0.001$ \\
4 adenomas & $1.4(0.98-2.2)$ & 0.06 \\
3 adenomas & $1.6(1.12-2.4)$ & 0.01 \\
2 adenomas & $1.4(0.96-2.09)$ & 0.07 \\
\hline
\end{tabular}

